\title{
A Study of Dosimetric Evaluation and Feasibility of Image Guided Intravascular Brachytherapy in Peripheral Arteries
}

\author{
Julien Bellec ${ }^{1}$, Jean-Pierre Manens ${ }^{1}$, Cemil Göksu ${ }^{2}$, Cécile Moisan ${ }^{3}$, and \\ Pascal Haigron ${ }^{2}$ \\ 1 CRLCC Eugène Marquis, CS 4422935042 Rennes, France \\ 2 LTSI, INSERM U642, Université de Rennes 1, 35042 Rennes, France \\ 3 Service de Chirurgie Vasculaire, CHRU, Rennes, France
}

\begin{abstract}
This work involves the conception of the experimental dosimetric testing setup of an image based procedure for intravascular brachytherapy by ${ }^{192}$ Ir seed in peripheral arteries. After making sure of the suitability of the basis dosimetry data used for dose calculation, the treatment sequence as a whole has been tested in a customized tissue-equivalent phantom. The method is based on the use of $\mathrm{CT}$ preoperative images and 2D intra-operative radiographs. Experimental dosimetric results agree with the planning and demonstrate the feasibility of the procedure.
\end{abstract}

\section{Introduction}

Intravascular Brachytherapy (IVB) constitutes a new therapeutic solution to avoid iterative redilations practiced after minimally invasive treatment of peripheral arterial stenoses. Following percutaneous transluminal angioplasty procedure, IVB aims at preventing intimal hyperplasia / restenosis. Its application to peripheral arteries by means of a high dose rate (HDR) ${ }^{192}$ Ir seed remains at a preliminary stage. Peripheral arteries present some specific features compared to coronary arteries as for their shape, geometrical characteristics (length, diameter, curvature) and the presence of surrounding anatomical structures. Based on AAPM TG 43/60 formalism [1,2] and a dedicated phantom model this work focuses on the dosimetric evaluation of the treatment sequence relating to the dose prescription with respect to the arterial wall as well as the precise localization of the seed from preoperative CT and intra-operative fluoroscopic images.

\section{Methods and Material}

First, in order to validate dose calculation, the TG 43 dosimetry parameters from our own ${ }^{192} \mathrm{Ir}$ seed have been experimentally evaluated (i.e. air kerma strength, dose rate constant, radial dose function and anisotropy function). Since no gold standard exists 
for dosimetry in IVB, different types of detectors have been analysed : a Nuclear Farmer cylindrical ionization chamber ; a PTW-Frieburg 31006 pinpoint chamber ; a PTW-Frieburg 60003 diamond detector and GR-100M Fimel LiF (Mg, Ti) thermoluminescent dosimeters (TLD). A water phantom from PTW-Frieburg and a polystyrene phantom have been used. The dosimetric evaluation of the whole treatment sequence (planning and tracking) has been carried out on a dedicated polystyrene phantom. In it, a simplified tubular model (straight cylindrical paths) has been digged. In order to measure the dose actually applied around the source train, a batch of TLD type GR$100 \mathrm{M}$ has been inserted into the phantom. To minimize uncertainties due to extremely large dose gradient near the ${ }^{192} \mathrm{Ir}$ source, these measurements have been conducted at a distance larger than $1 \mathrm{~cm}$. An intravascular navigation guidance method already presented in [3] has been implemented in order to locate the ${ }^{192} \operatorname{Ir}$ seed intra-operatively. The framework involves the simulation of 2D fluoroscopy images from both the CT data and the virtual $\mathrm{C}$-arm pose. Anatomical feature-based 2D/3D registration between a single $2 \mathrm{D}$ fluoroscopic view, reproduced from the planned pose, and the preoperative volume, is performed by minimizing the distance between the projection of a selected set of centerlines and the $2 \mathrm{D}$ vascular skeleton by means of a chamfer distance map.

\section{Results and Discussion}

Regarding the dose calculation validation, the maximum standard deviation between the experimental dosimetric parameters and those used in the treatment planning system is within $5 \%$ : the results are suitable with the recommandations [1]. In the same way, the measured dose around the source train during the simulated treatment are within $3 \%$ of the computed dose.

Therefore, the results demonstrate that IVB treatment sequence is valid in simplified experimental conditions (straight and centered source path). However, further inphantom investigations based on the artery model are necessary to complete the validation. An overall evaluation of the method should include both the curvature and the centering guide-catheter system.

\section{References}

1. Nath R, Anderson LL, Luxton G, Weaver KA, Williamson J Fand, Meigooni AS. Dosimetry of interstitial brachytherapy sources : Recommendations of the AAPM Radiation Therapy Committee Task Group No. 43. Med. Phys. 22: 209-234; 1995.

2. Nath R, Amols H, Coffey C, Jani S, Li Z, Schell MC, Soares C, Whiting J, Cole P, Crocker I, Schwartz R. Intravascular brachytherapy physics : Report of the AAPM Radiation Therapy Committee Task Group No. 60. Med. Phys. 26 (2): 119-152; 1999.

3. Göksu C, Haigron P, Zhang H, Soulas T, Le Certen G, Lucas A. 3D intraoperative localization for endovascular navigation guidance. In Surgetica'2002, Computer-Aided Medical Interventions: tools and applications, J Troccaz, Ph Merloz, eds., Sauramps Medical: 323329 ; 2002. 